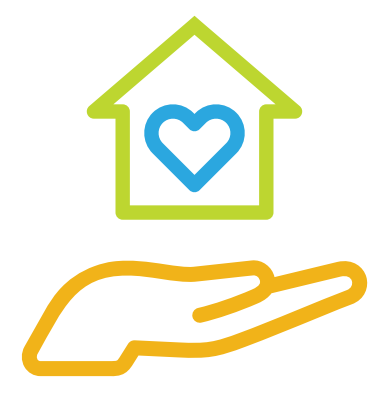

\title{
QUANDO O CUIDADO ENCONTRA A RUA: EXPERIÊNCIAS DE ENFRENTAMENTO À SÍFILIS COM A POPULAÇÃO EM SITUAÇÃO DE RUA
}

WHEN CARE MEETS THE STREET: EXPERIENCES OF COPING WITH SYPHILIS IN THE HOMELESS POPULATION

\begin{abstract}
Diego da Silva Medeiros
Sociólogo. Especialista em Saúde Pública. Mestre em Saúde Coletiva. Doutorando em Sociologia (UECE). Apoiador do Projeto Pesquisa Aplicada para Integração Inteligente Orientada ao Fortalecimento das Redes de Atenção para Resposta Rápida à Sífilis, Universidade Federal do Rio Grande do Norte. E-mail: diego.medeiros@lais.huol.ufrn.br.
\end{abstract}

\section{Louanne Aires Pereira}

Psicóloga. Especialista em Saúde da Família e Comunidade. Apoiadora do Projeto Pesquisa Aplicada para Integração Inteligente Orientada ao Fortalecimento das Redes de Atenção para Resposta Rápida à Sífilis,

Universidade Federal do Rio Grande do Norte. E-mail: louanne.aires@lais.huol.ufrn.br.

\section{Janete Romão dos Santos}

Enfermeira. Especialista em Gestão e Saúde. Especialista em Infectologia. Mestre em Saúde Pública. Voluntária do Grupo Espírita Casa da Sopa. Enfermeira do Hospital São José de Doenças Infecciosas.

E-mail: janeteromao25@gmail.com.

Mirleide de Brito Figueiredo

Assistente Social. Assessora Técnica da Área Técnica de IST, HIV/Aids e Hepatites Virais - Secretaria Municipal de Saúde de Fortaleza. E-mail: mirfigueiredo@gmail.com.

\section{Aline Rodrigues Feitoza}

Enfermeira. Especialista em Enfermagem Obstétrica e Enfermagem Forense. Mestre e doutora em

Enfermagem (UFC). Docente do Curso de Graduação em Enfermagem e do Mestrado em Tecnologia e Inovação em Enfermagem da Universidade de Fortaleza (UNIFOR). Assessora Técnica da Área Técnica de IST, HIV/Aids e Hepatites Virais - Secretaria Municipal de Saúde de Fortaleza. E-mail: alinerodfeitoza@gmail.com.

\section{Marcos Cavalcante Paiva}

Enfermeiro. Especialista em Educação Profissional da Área da Saúde. Especialista em Saúde Pública. Enfermeiro do Hospital São José de Doenças Infecciosas. Coordenador da Área Técnica de IST, HIV/Aids e Hepatites Virais - Secretaria Municipal de Saúde de Fortaleza. E-mail: olekmcp@gmail.com.

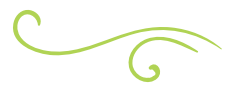

\section{RESUMO}

A população em situação de rua é prioritária para o enfrentamento da epidemia de sífilis.
Este estudo consiste em um relato de experiência das articulações do Projeto "Sífilis Não" no município de Fortaleza-Ce, para 
acesso da população em situação de rua à testagem rápida de sífilis. Para tanto, mobilizou instituições da saúde, da assistência social e da sociedade civil para a realização de duas campanhas extramuros de oferta de testes rápidos de sífilis. A intersetorialidade é fundamental para a resposta à sífilis. A primeira campanha ocorreu nas dependências de uma organização da sociedade civil; a segunda, num equipamento da assistência social para a população em situação de rua. Houve significativa adesão da população em situação de rua nas duas campanhas e a possibilidade de construção de vínculos no que diz respeito à sífilis. A efetividade de uma política pública de acesso dessa população à saúde requer que se observem as singularidades desse segmento, bem como os elementos sociais, políticos e econômicos que as atravessam. Os artifícios meramente biomédicos não são suficientes para impactar esse cenário. $O$ desafio é superar a dupla invisibilidade: a sífilis e a população em situação de rua. A manutenção das atividades de enfrentamento à sífilis requer um duplo tensionamento: das populações para as instituições e das instituições para as populações.

Palavras-chave: Sífilis; População em situação de rua; Teste rápido; Acesso à saúde; Organização da sociedade civil.

\section{ABSTRACT}

The homeless population is a priority for facing the syphilis epidemic. It is an experience report of the articulations of the "No Syphilis" Project in the city of Fortaleza to access rapid syphilis testing. To this end, the Project involved health, social assistance and civil society institutions carry out two extra-wall campaigns offering rapid syphilis tests. Intersectoriality is fundamental to the response to syphilis. The first campaign took place on the premises of a civil society organization, the second on social assistance equipment for the homeless population. There was significant adherence by the PSR in both campaigns and the possibility of building bonds concerning syphilis. The effectiveness of an accessible public policy of the homeless population requires observing the singularities of this segment, as well as the social, political and economic elements that go through them. Merely biomedical devices are not enough to impact this scenario. The challenge is to overcome the double invisibility: syphilis and homelessness. Maintaining activities to combat syphilis requires double tension: from population to institutions and from institutions to population.

Key-words: Syphilis; Homeless population; Rapid test, Access to health; Civil society organization.

\section{INTRODUÇÃO}

A sífilis segue causando morbidade e mortalidade em todo o mundo, embora as estratégias biomédicas de prevenção, diagnóstico e tratamento estejam disponíveis para o controle dessa infecção sexualmente transmissível (IST). Os casos têm recrudescido em países de alta renda e permanecido endêmicos em países de baixa e média renda, como o Brasil (KOJIMA; KLAUSNER, 2018). A cada ano, cerca de seis milhões de novos casos de sífilis em pessoas com idades entre 15 e 49 anos são identificados, principalmente concentrados em populações específicas (STEVENS et al., 2015).

Em 2019, foram registrados 152.915 novos casos de sífilis adquirida no Brasil. Entre os anos de 2015 e 2018, houve aumento na taxa de detecção em sífilis adquirida, que saltou de 34,1 casos para 76,2 casos por 100.000 habitantes, respectivamente. Ainda em 2019, a maior parte das notificações de indivíduos com sífilis adquirida ocorreu em 36,2\% na faixa etária de 20 e 29 anos e 21,8\% na faixa etária de 30 a 39 anos. Em relação ao gênero, foram diagnosticados $41,1 \%$ de homens e $58,9 \%$ de mulheres, e, 
destas, $47,1 \%$ foram notificadas como sífilis adquirida e $52,9 \%$ como sífilis em gestantes, sugerindo o maior número de casos em mulheres devido à oferta da testagem no protocolo do pré-natal. As pessoas pardas $(38,1 \%)$ e pretas $(10,2 \%)$ compreendem $48,3 \%$ dos casos de sífilis adquirida, enquanto as brancas $35,3 \%$ e $15,1 \%$ de ignorados (BRASIL, 2020). Esses dados revelam não só o aprimoramento da vigilância epidemiológica mas também uma epidemia ascendente e insistente, principalmente quando se consideram as singularidades dos indivíduos, as regiões e os territórios.

O estado do Ceará apresenta dinâmica semelhante. Em 2019, foram notificados 3.169 casos de sífilis adquirida. Entre os anos de 2015 e 2019, a taxa de detecção oscilou de 7,6 para 34,7 casos por 100.000 habitantes, respectivamente. A Superintendência Regional de Saúde (SRS) de Fortaleza é responsável pela detecção de $71,6 \%$ dos casos (CEARÁ, 2020).

A epidemia de sífilis apresenta sinais de concentração em grupos populacionais específicos. O Departamento de Doenças de Condições Crônicas e Infecções Sexualmente Transmissíveis (DCCl) do Ministério da Saúde (MS), alinhado à Organização Mundial de Saúde (OMS), nomeia populações-chave (key populations) aquelas atingidas por ISTs de forma desproporcional e desigual em comparação à população geral, tanto no aspecto epidemiológico quanto em relação ao acesso à saúde. São elas: homens que fazem sexo com homens (HSH), usuários de álcool e outras drogas, pessoas privadas de liberdade, trabalhadores do sexo e transgêneros (BRASIL, 2018).

Ainda no aspecto conceitual, nomeiam-se de "populações prioritárias" as que, associadas às populações-chave, apresentam vulnerabilidades aumentadas devido à situação de vida ou a contextos históricos, sociais e estruturais, a saber: jovens, população preta, indígenas e pessoas em situação de rua. Importa dizer, todavia, que não há uma identidade pura para esse sujeito. $O$ indivíduo pode ser atravessado por mais de uma condição, isto é, um HSH pode estar em situação de rua e abusar de álcool e outras substâncias psicoativas, configurando aquilo que Richard Parker (2000) denominou de "sinergia de vulnerabilidades". Todos os segmentos populacionais denominados "chaves" ou "prioritários" devem ser observados como potencialmente transversais (BRASIL, 2018).

A população em situação de rua (PSR) caracteriza-se pela pobreza extrema; vínculos familiares interrompidos ou fragilizados; inexistência de moradia convencional regular; usuários de logradouros públicos ou áreas degradadas como espaço de moradia e de sustento, de forma temporária ou permanente; usuários de unidades de acolhimento para pernoite temporário ou como moradia provisória. Segundo censo realizado em 2014 pela Secretaria Municipal dos Direitos Humanos e Desenvolvimento Social (SDHDS), o município de Fortaleza possuía 1.718 indivíduos em situação de rua; destes, $74,6 \%$ estavam na faixa etária de 19 e 44 anos; 83,5\% identificaram-se como sendo do sexo masculino; e $79,1 \%$ com ensino fundamental ou inferior. Essa população está concentrada, em sua maioria, no território da Secretaria Regional II (SR II), nos bairros do Centro e Beira Mar (FORTALEZA, 2018).

O censo revelou, ainda, que $79,8 \%$ faziam uso de algum tipo de droga; $52,8 \%$ estavam há pelo menos dois anos morando na rua; $38,35 \%$ não utilizavam serviços públicos; 37,2\% tinham problemas de saúde; $45,6 \%$ sofreram violência na rua; $46,1 \%$ ganhavam, em média, abaixo de 100 reais por semana (FORTALEZA, 2018). Uma característica marcante das PSR é a heterogeneidade, de modo que uma análise acurada requer a observância do contexto cultural, social, econômico, histórico, comunitário e pessoal (ESMERALDO FILHO, 2010).

A PSR estabelece vínculos mais facilmente com os equipamentos da assistência 
social e das organizações da sociedade civil em relação às unidades de saúde (SANTOS, 2019). O acolhimento desse segmento da população nas unidades da atenção primária é limitado, em virtude das barreiras impostas pela estrutura e pelos profissionais de saúde, tais como preconceito, desrespeito, mau atendimento e insatisfação dos usuários (ESMERALDINO FILHO, 2010; SANTOS, 2019).

Pesquisas realizadas com a população em situação de rua abrigada em Goiânia apontaram prevalência de sífilis bastante elevada, demonstrando a importância da intervenção educacional, do aprimoramento dos programas de redução de riscos, do desenvolvimento de estratégias de prevenção e controle, incluindo aconselhamento e testagem, bem como o oferecimento de tratamento para ISTs nos cenários de rua e abrigos temporários e/ou permanentes (PINHEIRO, 2017; BARROS et al., 2018).

Investigação realizada no município de São Paulo afirma que a PSR pode ser receptiva à oferta de teste rápido para a sífilis, bem como ao tratamento dos casos reagentes. No entanto, ações extramuros sistemáticas de oferta de testes não são observadas. Essas atividades são fundamentais para proporcionar o acesso à saúde de uma população historicamente invisibilizada nas unidades de saúde. Políticas de rastreamento da sífilis e outras ISTs que não considerem as populações assintomáticas de difícil acesso, como a PSR, podem acarretar perda de oportunidade de redução significativa das infecções (PINTO et al., 2014).

O Projeto "Sífilis Não", por meio dos apoiadores articulados prioritariamente à Secretaria Municipal de Saúde (SMS), tem a intenção de intervir no acesso à saúde da PSR, população prioritária para a epidemia de sífilis, tecendo redes com ampla capilarização, envolvendo outras secretarias e organizações da sociedade civil e observando as singularidades do território vivo para além da perspectiva biopolítica de controle dos corpos e do cuidado meramente protocolar (FRANCO, 2013; MERHY et al., 2014).

O objetivo deste estudo consiste em fomentar experiências de atividades extramuros que proporcionem à PSR acesso à saúde por meio da testagem rápida de sífilis. Essas experiências, a um só tempo, consideraram a formação e atualização sobre sífilis (aspectos clínicos, epidemiológicos e sociais) para as (os) agentes envolvidas (os) e para as singularidades desse segmento da população na construção de ações com a temática das infecções sexualmente transmissíveis (ISTs).

Para esse intento, a rede viva de cuidado constituída (MERHY et al., 2014) contou com: apoiadores do Projeto "Sífilis Não", Área Técnica de IST, HIV/Aids e hepatites virais, Coordenadoria Regional de Saúde II, Estratégia Fique Sabendo Jovem, Liga Acadêmica de Estudos e Pesquisas do Vírus da Imunodeficiência Humana (LAVIH) da Universidade de Fortaleza (UNIFOR), Grupo Espírita Casa da Sopa, Unidade de Atendimento Primário à Saúde Paulo Marcelo (UAPS Paulo Marcelo), Consultório na Rua e Centro de Convivência da População em Situação de Rua.

\section{MÉTODO}

Trata-se de um relato de experiência dos apoiadores do Projeto "Sífilis Não" no município de Fortaleza para articulação de uma rede viva de cuidado que compreende instituições para fomento de ações extramuros de ofertas de testes rápidos para sífilis junto à população em situação de rua (PSR).

As articulações resultaram em duas ações: a primeira realizada nas dependências de uma organização da sociedade civil (OSC) chamada Grupo Espírita Casa da Sopa (GECS), no dia 21 de outubro de 2019; a segunda no Centro de Convivência para a População em Situação de Rua (CCPSR), equipamento da Secretaria de Direitos Humanos e Desenvolvimento Social 
(SDHDS), no dia 13 de outubro de 2020. As duas instituições estão localizadas no centro do município de Fortaleza, espaço preferencial da PSR (FORTALEZA, 2018), e acolhem, a seu modo, essa população. Nas ações realizadas, os apoiadores do Projeto "Sífilis Não" estiveram associados à Área Técnica de IST, HIV/Aids e hepatites virais da SMS e à Coordenadoria Regional de Saúde II (CORES II) da Secretaria Regional II (SR II), ambas disponibilizando os insumos necessários para a realização das atividades.

O GECS é uma organização alinhada aos preceitos espiritistas, que atua no centro de Fortaleza há 25 anos, com atividades específicas em cada dia da semana. O trabalho consiste em acolher a PSR que deseja se alimentar, tomar um banho, estudar o evangelho, acessar vestimentas e outros objetos disponibilizados pela organização, além de atendimento ambulatorial de saúde.

Aarticulaçãofoitecida coma Coordenação de Saúde da instituição que nos acolheu em reunião preliminar de planejamento da ação. Participaram da atividade os apoiadores do Projeto "Sífilis Não"; técnicas (os) da Área Técnica de IST, HIV/Aids e hepatites virais; acadêmicas(os) da LAVIH-UNIFOR; membros da Estratégia Fique Sabendo Jovem; e voluntárias(os) do GECS.

O CCPSR é um equipamento da SDHDS que funciona de segunda a sexta, no horário comercial, e está localizado no centro de Fortaleza, proporcionando espaço, durante o dia, para atividades de socialização, fortalecimento de vínculos interpessoais e familiares e cuidados básicos com alimentação, higiene pessoal e lavanderia. A capacidade é de 200 pessoas por dia, entre homens, mulheres adultas e famílias em situação de rua. A ação foi articulada pela CORES II e profissionais de saúde do Consultório na Rua, os quais têm por base a UAPS Paulo Marcelo, e contou com o apoio do Projeto "Ś́filis Não" e da Área Técnica de IST, HIV/Aids e hepatites virais.

\section{RESULTADOS E DISCUSSÃO}

Os apoiadores do Projeto "Sífilis Não" têm por atividade observar os territórios, as potencialidades e os obstáculos para ações de construção de redes vivas de cuidado e incremento do acesso à saúde das populações-chave e prioritárias para a epidemia de sífilis. Em relação à PSR no município de Fortaleza, os territórios são amplamente conhecidos pelas políticas públicas da assistência social, das organizações da sociedade civil (OSC) e da saúde, inclusive as instituições estão sediadas no próprio território. A Área Técnica de IST, HIV/Aids e hepatites virais, com sede no centro da cidade e parceira intrínseca do Projeto "Sífilis Não", já monitora e atua no território.

O nosso papel, portanto, é auxiliar a gestão municipal no fomento das redes vivas de cuidado, e, para tanto, são decididas em conjunto as potenciais parcerias que lidam cotidianamente com a PSR. O município de Fortaleza possui divisão administrativa em seis Secretarias Regionais (SR) e cada SR possui uma Coordenadoria Regional de Saúde (CORES). Assim, o território do centro de Fortaleza está sob responsabilidade sanitária da CORES II, que participa ativamente do planejamento e articulação das ações.

\section{GRUPO ESPÍRITA CASA DA SOPA (GECS)}

O GECS é uma organização da sociedade civil de longeva atuação no território do centro de Fortaleza, com atividade destacada no acolhimento à PSR há mais de duas décadas. A instituição já realizou atividades pontuais de oferta de testes rápidos junto à Área Técnica de IST, HIV/Aids e hepatites virais. $\bigcirc$ desafio atual, portanto, é estabelecer um fluxo sistemático.

O GECS é composto por voluntárias(os) que realizam o trabalho semanal. Não há trabalhadoras(es) formais remuneradas(os) e a instituição conta com uma Coordenação de 
Saúde composta de profissionais de saúde igualmente voluntárias(os). A ação relatada teve articulação com a Coordenação de Saúde do GECS em reuniões prévias realizadas na sede da instituição, encontros que são marcados por atualizações sobre a epidemia de sífilis e planejamentos das ações segundo o espaço físico e disponibilidade das(os) agentes envolvidas(os).

As atividades ordinárias do GECS se iniciam, geralmente, no final da tarde. Em cada dia da semana há uma atividade distinta. Às segundas-feiras, em específico, há O acoIhimento da PSR para banho, alimentação, estudo do evangelho com música de harmonização e atendimentos de saúde, caso necessitem. Esse foi o dia escolhido para ação disparada pelo Projeto "Sífilis Não" em parceria com a Estratégia Fique Sabendo Jovem e a Liga Acadêmica de Estudos e Pesquisas do Vírus da Imunodeficiência Humana (LAVIH) da Universidade de Fortaleza (UNIFOR).

As(os) profissionais de saúde, acadêmicas(os) e voluntárias(os) do GECS estavam organizadas(os) em pontos de atuação para a oferta do teste rápido para sífilis, tais como o acolhimento daquelas(es) que desejavam fazer o teste em lista de interesse; condução para a sala fechada onde ocorria o pré-aconselhamento; em seguida, para sala onde se realizaria a coleta do material biológico; e, por fim, até a sala fechada para entrega do resultado. A organização da atividade estava atenta para garantir a realização do teste de acordo com a chegada das(os) usuárias(os) e com o máximo de discrição e sigilo possível. Essa tecnologia de oferta de teste extramuros é bastante utilizada pela Área Técnica de IST, HIV/Aids e hepatites virais, que possui expertise consolidada.

$\mathrm{Na}$ ocasião, 27 pessoas realizaram o teste rápido treponêmico para sífilis com oito resultados reagentes. Esses indivíduos foram referenciados para a unidade básica de saúde previamente comunicada da ação no GECS. Avaliamos como significativa a adesão das pessoas à campanha, em consonância com estudo semelhante relativo à recepção dos testes junto às PSR (PINTO et al., 2014). A limitação, no entanto, consiste na incógnita relativa ao encaminhamento das pessoas com resultado reagente, devido às conhecidas barreiras de acesso na atenção primária. Essa limitação do seguimento poderia ser dirimida caso estivesse disponível a administração da primeira dose de penicilina benzatina na ocasião da campanha, questão que, todavia, está sendo amplamente discutida nos espaços colegiados do Projeto "Sífilis Não" no município de Fortaleza, para aplicação em curto prazo.

A campanha disparou processos de visibilidade da epidemia de sífilis entre as(os) voluntárias(os) e as(os) usuárias(os) do GECS. Na ocasião, foram observados: participação de pessoas receosas da realização dos testes, mas encorajadas(os) pelas(os) colegas da rua; os aspectos informativos e educativos da campanha sobre práticas sexuais mais seguras e a importância do diagnóstico oportuno; a oportunidade de estar em contato com profissionais de saúde para orientações em ambiente discreto e sigiloso; e, por fim, a referência da instituição como espaço de cuidado (há relatos das(os) voluntárias(os) sobre sondagens, meses depois em relação à oferta de testes). $O$ planejamento consistia em campanhas com frequência semestral, mas sofreu descontinuidade devido à emergência da pandemia de covid-19. As articulações estão sendo retomadas com a participação da Rede Nacional de Médicas e Médicos Populares para os próximos meses.

\section{CENTRO DE CONVIVÊNCIA PARA A POPULAÇÃO EM SITUAÇÃO DE RUAA (CCPSR)}

Os equipamentos da assistência social para a população em situação de rua, sob a responsabilidade da Secretaria de Direitos Humanos e Desenvolvimento Social (SDHDS), são estratégicos para ações sistemáticas de 
enfrentamento à sífilis. Segundo Santos (2019), a PSR tem mais familiaridade com os equipamentos da assistência social do que com as unidades de saúde. Os serviços da assistência social para a população adulta em situação de rua no município de Fortaleza contam com um Centro de Convivência, dois Centros Pop, duas Pousadas Sociais, uma Casa de Passagem, dois Abrigos Institucionais (um para homens, outro para mulheres e famílias) e um Refeitório Social.

O CCPSR está a duas quadras da UAPS Paulo Marcelo, ambos localizados no centro de Fortaleza. O Consultório na Rua (CR), com base na UAPS, já realiza, pontualmente, ações de saúde no CCPSR. Motivada pelo mês de outubro de enfrentamento à sífilis e provocada pelos apoiadores do Projeto "Sífilis Não", a CORES II mobilizou o CR para ações no âmbito da sífilis, que foram prontamente acatadas pela equipe. $\mathrm{O} C R$ entrou em contato com a coordenação do CCPSR, que disponibilizou o espaço físico para a realização da campanha de testagem de sífilis. Estiveram envolvidas(os) na ação as(os) profissionais do CCPSR, de saúde do CR e da UAPS Paulo Marcelo, além dos apoiadores do Projeto "Sífilis Não".

O CCPSR é um espaço de acolhimento durante $\mathrm{O}$ dia que disponibiliza cuidados básicos (banho, alimentação e lavanderia), atendimento sobre direitos sociais, desenvolvimento de atividades de sociabilidades para fortalecimentos de vínculos e construção de projetos de vida. O espaço mais ao fundo do prédio contém uma televisão com cadeiras e mesas dispostas para atividades de lazer das(os) usuárias(os) e um espaço livre onde há uma piscina aterrada. Como não havia salas disponíveis para a ação, mais ao fundo do prédio, posicionamos mesas e cadeiras para o fluxo da oferta de testes rápidos.

No intuito de garantir a discrição e o sigilo, mantemos distância de uma mesa para outra. Havia, então, três pontos para ação: duas mesas para aconselhamento pré-teste e entrega dos resultados; um conjunto de mesas para a coleta de material biológico.
As(os) usuárias(os) que estavam nesse ambiente com a televisão manifestavam interesse em participar da testagem e um profissional do CCPSR registrava cada um. Segundo a ordem da manifestação do interesse, as(os) usuárias(os) eram conduzidas(os) para as mesas de pré-aconselhamento, e, em seguida, seguiam para o conjunto de mesas para coleta do material biológico. Depois de realizado o exame, retornavam à primeira mesa para a entrega do resultado.

$\mathrm{Na}$ ocasião, 23 pessoas realizaram o teste rápido treponêmico para sífilis e nenhum resultado reagente. As(os) usuárias(os) que realizaram a testagem tiveram orientações sobre promoção, prevenção, sintomas de IST e outros agravos, além de tratar sobre o acesso ao serviço pelas profissionais de saúde participantes da ação que atuam na própria UAPS Paulo Marcelo, possibilitando estreitamento do vínculo. A participação do $C R$ e da UAPS Paulo Marcelo permite que as(os) profissionais e usuárias(os) do CCPSR conheçam as profissionais de saúde e facilitem o acesso à atenção primária, ainda comprometido por uma série de barreiras, estas, inclusive, descritas aos apoiadores do Projeto "Sífilis Não" no momento do pré-aconselhamento por pessoas que fariam a testagem.

A campanha de testagem para sífilis aproximou os apoiadores do Projeto "Sífilis Não" da coordenação do CCPSR que, por sua vez, aproximou-nos da gestão central dos equipamentos da assistência social para a população em situação de rua da SDHDS. A ideia é disparar um processo de formação sobre sífilis para as(os) profissionais que atuam naqueles equipamentos, no intuito de que se sintam seguras(os) em abordar o tema; disponibilizar bancos de preservativos em parceria com a Área Técnica de IST, HIV/ Aids e hepatites virais para todos os equipamentos; e identificar possíveis profissionais de saúde atuantes nesses equipamentos para capacitação e realização da testagem rápida para sífilis.

A participação do CR nessas atividades é de fundamental importância; no entanto, o 
funcionamento restrito ao horário comercial é um ponto limitador, o que impediu, por exemplo, a participação na campanha de oferta de testes no GECS, pois a ação ocorreu no período noturno, momento propício para a aproximação da PSR que não está abrigada em equipamentos da assistência social. A expectativa pós-campanha é a referência sistemática e estreita do CCPSR com o CR e a UAPS Paulo Marcelo para as questões que dizem respeito à sífilis.

\section{CONSIDERAÇÕES FINAIS}

O grande desafio do Projeto "Sífilis Não" é a visibilidade da epidemia de sífilis nas ações que se engendram nos espaços da saúde. Compreender a sífilis para além dos exames do pré-natal, ainda frágeis, requer que desloquemos a sexualidade da sua faceta meramente reprodutiva e a posicionemos na vida integral das pessoas. Essas ações não podem prescindir do contato com as populações-chave e prioritárias, sob pena de não impactarem verdadeiramente a epidemia de sífilis.

Há uma segunda invisibilidade: a população em situação de rua. Trabalhamos com uma dupla invisibilidade que incrementa a vulnerabilidade para as ISTs. O movimento engendrado pelo Projeto "Sífilis Não" é de observação do território vivo e posterior identificação de instituições e sujeitos que estejam próximos da população em situação de rua, para, em conjunto, viabilizar ações que promovam a saúde, a prevenção, o diagnóstico oportuno e o tratamento. Assim, além do campo da saúde, que precisa estar mobilizado, é mister que se envolvam as organizações da sociedade civil e os equipamentos da assistência social que estão no cotidiano das populações em situação de rua.

A manutenção das atividades que consideram a sífilis requer um duplo tensionamento: aquele que vem da base, da população, seja individual ou coletivamente, junto às instituições (sejam elas de saúde, assistência social ou educação); e, o outro, aquele que vem das instituições para a base. Na medida em que um desses polos está fragilizado, o outro deve empreender esforços para manter a visibilidade de uma epidemia que requer mais do que insumos biomédicos.

\section{AGRADECIMENTOS}

A todas as instituições citadas neste relato de experiência, bem como às(aos) profissionais de saúde, às(aos) profissionais das instituições da assistência social e às(aos) voluntárias(os) das organizações da sociedade civil, que se empenharam nas ações de enfrentamento à sífilis. 


\section{REFERÊNCIAS}

BARROS, C. V. L. et al. Bio-behavioral survey of syphilis in homeless men in Central Brazil: a cross-sectional study. Cadernos de Saúde Pública, v. 34, n. 6, jun. 2018.

BRASIL. Ministério da Saúde. Secretaria de Vigilância em Saúde. Departamento de Vigilância, Prevenção e Controle das Infecções Sexualmente Transmissíveis. Agenda estratégica para ampliação do acesso e cuidado integral das populações-chave em HIV, hepatites virais e outras infecções sexualmente transmissíveis. Brasília/DF: Ministério da Saúde, 2018.

BRASIL. Ministério da Saúde. Secretaria de Vigilância em Saúde. Departamento de Doenças Crônicas e Infecções Sexualmente Transmissíveis. Boletim Epidemiológico Especial - Sífilis. Brasília/DF: Ministério da Saúde, 2020.

CEARÁ. Secretaria da Saúde do Estado do Ceará. Secretaria Executiva de Vigilância em Saúde e Regulação. Boletim Epidemiológico de Sífilis, n. 04. Fortaleza/CE: Secretaria de Saúde, 2020.

ESMERALDO FILHO, C. E. Necessidades de saúde dos moradores de rua: desafios para as políticas sociais do município de Fortaleza-CE. 2010. 159f. Dissertação (Mestrado Acadêmico em Saúde Pública) - Centro de Ciências da Saúde, Universidade Estadual do Ceará, Fortaleza, 2010.

FORTALEZA. Secretaria Municipal dos Direitos Humanos e Desenvolvimento Social. Cartilha de Políticas Públicas para População em Situação de Rua de Fortaleza. Fortaleza/CE: SDHDS, 2018.

FRANCO, T. B.; MERHY, E. E. Trabalho, produção do cuidado e subjetividade em saúde. São Paulo: Hucitec, 2013.

KOJIMA N.; KLAUSNER J.D. An Update on the Global Epidemiology of Syphilis. Current Epidemiology Reports, v. 5, n. 1, p. 24-38, mar. 2018.

MERHY, E. E. et al. Redes vivas: multiplicidades girando as existências, sinais da rua; implicações para a produção do cuidado e a produção do conhecimento em saúde. Divulgação em Saúde para Debate, Rio de Janeiro, n. 52, p. 153-164, out. 2014.

PARKER, R. Na contramão da Aids: sexualidade, intervenção, política. Rio de Janeiro: ABIA; São Paulo: Editora 34, 2000.

PINHEIRO, R. S. Pessoas em situação de rua abrigadas: uma população-chave na epidemiologia da sífilis e infecção pelo HIV em Goiânia-Goiás. 2017. 195f. Tese (Doutorado em Enfermagem) - Universidade Federal de Goiás, Goiânia, 2017.

PINTO, V. M. et al. Prevalência de Sífilis e fatores associados a população em situação de rua de São Paulo, Brasil, com utilização de Teste Rápido. Revista Brasileira de Epidemiologia, São Paulo, v. 17, n. 2, p. 341-354, jun. 2014. 
SANTOS, J. R. Viver na rua e a atuação das redes em saúde. 2019. 88f. Dissertação (Mestrado Acadêmico em Saúde Pública) - Faculdade de Medicina, Departamento de Saúde Comunitária, Universidade Federal do Ceará, Fortaleza, 2019.

STEVENS, G. et al. Global Estimates of the Prevalence and Incidence of Four Curable Sexually Transmitted Infections in 2012 Based on Systematic Review and Global Reporting. PLoS One, v. 10, n. 12, dez. 2015. 